\title{
Impactos da implantação do sistema informatizado para processo de seleção do PNAES no IFTO Campus Porto Nacional
}

\author{
Vanessa A. Corado, Mayara K. F. Cabral, Ismael A. Matos, Paulo César de Sousa \\ Patrício, Mara Cleide O. dos Santos
}

\author{
Instituto Federal de Educação, Ciência e Tecnologia do \\ Estado do Tocantins (IFTO) - Campus de Porto Nacional \\ 77500-000 - Porto Nacional - TO - Brasil \\ vanessaa.corado@gmail.com, mayarakflifto.edu.br, \\ ismael.matos@ifto.edu.br, paulo.patricio@ifto.edu.br, \\ maracleidedifto.edu.br
}

\begin{abstract}
This article discusses the impacts of the implementation of a web system designed to assist the distribution of funds from the National Student Assistance Program (PNAES) among students at the Federal Institute of Education, Science and Technology of Tocantins, Campus Porto Nacional IFTO. The analysis started from the perspective of own system users (candidates aid) and the local committee of student assistance (responsible for the selection process). The research aims to present a reflection on the positive and negative aspects of the implementation of systems for use by low-income students.
\end{abstract}

Resumo. Este artigo discute os impactos da implantação de um sistema web criado para auxiliar a distribuição dos recursos do Programa Nacional de Assistência Estudantil(PNAES) entre os estudantes no Instituto Federal de Educação, Ciência e Tecnologia do Tocantins, Campus Porto Nacional IFTO. A análise partiu da ótica dos próprios usuários do sistema (candidatos aos auxílios) e da comissão local da assistência estudantil (responsável pelo processo seletivo). A pesquisa busca apresentar uma reflexão sobre os aspectos positivos e negativos da implantação de sistemas para uso de estudantes de baixa renda.

\section{Introdução}

O PNAES é um programa nacional de assistência estudantil voltado para o amparo dos alunos em situação de vulnerabilidade social e econômica em instituições de ensino superior e Institutos Federais. É um meio de contribuição para o combate à evasão e ao fracasso escolar nas Instituições Federais de Ensino. A política de assistência estudantil revelou-se, ao longo do tempo, como uma maneira de garantir a permanência dos estudantes com condições socioeconômicas desfavoráveis no ensino superior (ASSIS, 2013). 
O PNAES tem se caracterizado pela tentativa de expandir este direito fundamental e buscar construir uma universidade que se estabeleça como a mediação da cidadania e da democracia na sociedade (SEVERINO, 2008).

Um dos desafios dentro do IFTO, apontado pela Comissão Local de Assistência Estudantil em 2013, foi a informatização do processo de seleção dos estudantes beneficiários, com o objetivo de facilitar a operacionalização e a análise sócioeconômica necessária para a distribuição das bolsas sociais. Como diz GUARNICA (2009):

\begin{abstract}
A utilização do conhecimento gerado nas universidades brasileiras representa rica fonte de informação e capacitação para o desenvolvimento de novas tecnologias, resultando no fato de que a transferência de tecnologia entre universidade e setor produtivo consiste em um caminho alternativo e complementar para o alcance de um patamar tecnológico superior das empresas brasileiras. (GUARNICA et all, 2009, pg 624).
\end{abstract}

Segundo o autor, as universidades brasileiras são capacitadas para o desenvolvimento de sistemas. Baseando-se nesta visão e como condição norteadora desta pesquisa, implementou-se um sistema web, até então, não existente na rede IFTO, para gerenciamento das bolsas do PNAES, chamado GD-PNAES (Gerenciamento de Distribuição do Programa Nacional de Assistência Estudantil).

Portanto, este trabalho, tem como objetivo descrever os resultados da pesquisa sobre os impactos da implantação do sistema web criado para auxiliar a distribuição dos recursos do Programa Nacional de Assistência Estudantil entre os estudantes no Instituto Federal de Educação, Ciência e Tecnologia do Tocantins, Campus Porto Nacional. A análise partiu da ótica dos próprios usuários do sistema (candidatos aos auxílios) e da comissão local da assistência estudantil (responsável pelo processo seletivo). A pesquisa busca apresentar uma análise das dificuldades de implantação de sistemas computadorizados para uso de estudantes de baixa renda.

\title{
2. O PNAES no IFTO
}

O PNAES criado por meio do Decreto Presidencial $n^{\circ} 7.234 / 2010$ é regido no IFTO pelo Regulamento do Programa de Assistência Estudantil, aprovado pela Resolução $\mathrm{n}^{\circ}$ 04/2011/CONSUP/IFTO, de 30 de junho de 2011 e alterado pela Resolução $\mathrm{n}^{\circ}$ 22/2014/CONSUP/IFTO, de 8 de agosto de 2014.

Segundo o Portal Brasil (2014) mais de um bilhão foram investidos em assistência estudantil a alunos de ensino superior federal nos últimos cinco anos, e que no ano de 2013, o volume destinado ao programa PNAES quadruplicou - passou de $\mathrm{R} \$ 126,3$ milhões para R \$ 603,8 milhões (Gráfico 1). No IFTO, Campus Porto Nacional, foram investidos no ano de 2014 cerca de 340 mil reais (IFTO, 2014). 


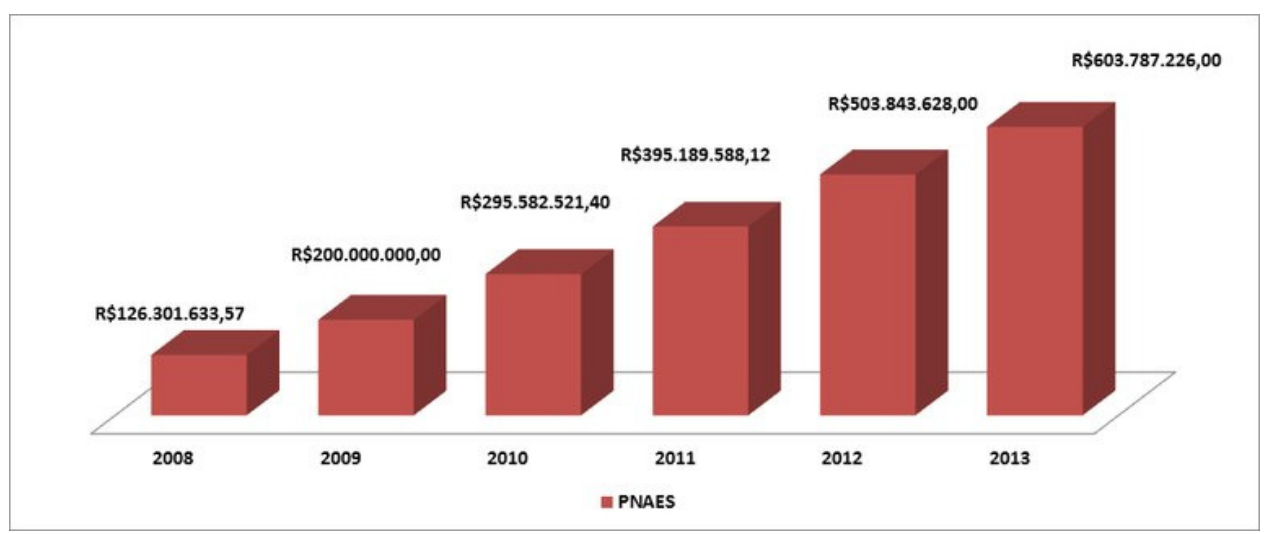

Gráfico 1: Demonstrativo dos gastos do governo de 2008 á 2013 com PNAES Fonte: Brasil.gov.br.

Priorizando os alunos em situação de vulnerabilidade socioeconômica, o processo seletivo para distribuição do recurso destinado ao programa é concedido por meio de bolsas sociais. As bolsas são classificadas de acordo com o indicador situacional do estudante candidato a bolsa (Tabela 1):

Tabela 1: Auxílios ofertados no campus e indicador situacional.

\begin{tabular}{|l|l|}
\hline Descrição da Bolsa & \multicolumn{1}{|c|}{ Indicador Situacional } \\
\hline Bolsa Atleta & $\begin{array}{l}\text { Alunos atletas em treinamento que representam o IFTO } \\
\text { diferentes modalidades esportivas. }\end{array}$ \\
\hline $\begin{array}{l}\text { Bolsa Formação } \\
\text { Profissional } \\
\text { (Estudante } \\
\text { Colaborador) }\end{array}$ & $\begin{array}{l}\text { Alunos sem vínculo empregatício e com disponibilidade para } \\
\text { desenvolverem atividades nos setores administrativos do IFTO. }\end{array}$ \\
\hline Auxílio Moradia & $\begin{array}{l}\text { Alunos que moram de aluguel e não possui familiares no } \\
\text { município de localização do IFTO. }\end{array}$ \\
\hline $\begin{array}{l}\text { Auxilio Creche } \\
\text { Auxilio Transporte } \\
\text { Urbano }\end{array}$ & $\begin{array}{l}\text { Alunos que são pais e que se encontram com dificuldade para } \\
\text { conciliar estudos e o cuidado com os filhos. }\end{array}$ \\
\hline $\begin{array}{l}\text { Para os alunos que residem em localização distante do IFTO, perímetro urbano, e que tem dificuldades de } \\
\text { locomoção, devido à falta de recurso financeiro. }\end{array}$ \\
\hline $\begin{array}{l}\text { Intermunicipal ou } \\
\text { Rural }\end{array}$ & $\begin{array}{l}\text { Para alunos que residem em outra cidade ou na área rural e que } \\
\text { tem dificuldades de locomoção, devido à falta de recurso } \\
\text { financeiro. }\end{array}$ \\
\hline Bolsa Emergencial & $\begin{array}{l}\text { Estudantes que passam por situação de desemprego, problemas } \\
\text { de saúde e outros motivos que o enquadrem em situação de } \\
\text { emergência. }\end{array}$ \\
\hline
\end{tabular}

\section{Metodologia}

Esta pesquisa foi organizada em seis etapas: i)Levantamento de materiais bibliográficos para leitura e embasamento conceitual; ii)Levantamento de softwares similares; iii) 
Desenvolvimento do sistema; e iv) Implantação do sistema; v) Avaliação do sistema nas visão dos usuários e vi) Análise dos resultados por meio de textos e tabelas.

Com a finalidade de identificar softwares similares e já existentes, realizou-se uma pesquisa através do buscador Google, por meio das palavras-chaves: "PNAES", "Software". Não foi constatado nenhum sistema que pudesse ser utilizado para gerenciamento de bolsas que fosse gratuito e se adequasse os requisitos da Comissão Local de Assistência Estudantil para distribuição dos recursos do PNAES. Foram identificados apenas notícias de processos seletivos tradicionais e formulários a serem impressos e preenchidos manualmente. Realizado uma pesquisa entre os campi do IFTO, onde foi identificado que até o ano de 2013, quando iniciou-se o desenvolvimento do sistema, em todos os campi o processo de seleção dos estudantes era feito manualmente através de formulários impressos.

O sistema foi desenvolvido utilizando as linguagens de programação PHP (HyperText PreProcessor), HTML (HyperText Markup Language), CSS (Cascading Style Sheets) e base de dados MySql(My Structured Query Language). O sistema web implementado possui funcionalidades descritas a partir do EAP (Estrutura Analítica do Projeto) (Figura 1) descrita pelo PMI como sendo:

De composição hierárquica orientada às entregas do trabalho a ser executada pela equipe para atingir os objetivos do projeto e criar as entregas requisitadas, sendo que cada nível descendente da EAP representa uma definição gradualmente mais detalhada da definição do trabalho do projeto (PMI, 2008, p.116).

O GD-PNAES foi construído de forma modular, possuindo basicamente dois módulos principais: Módulo Aluno e Módulo Administrador (Figura 1)

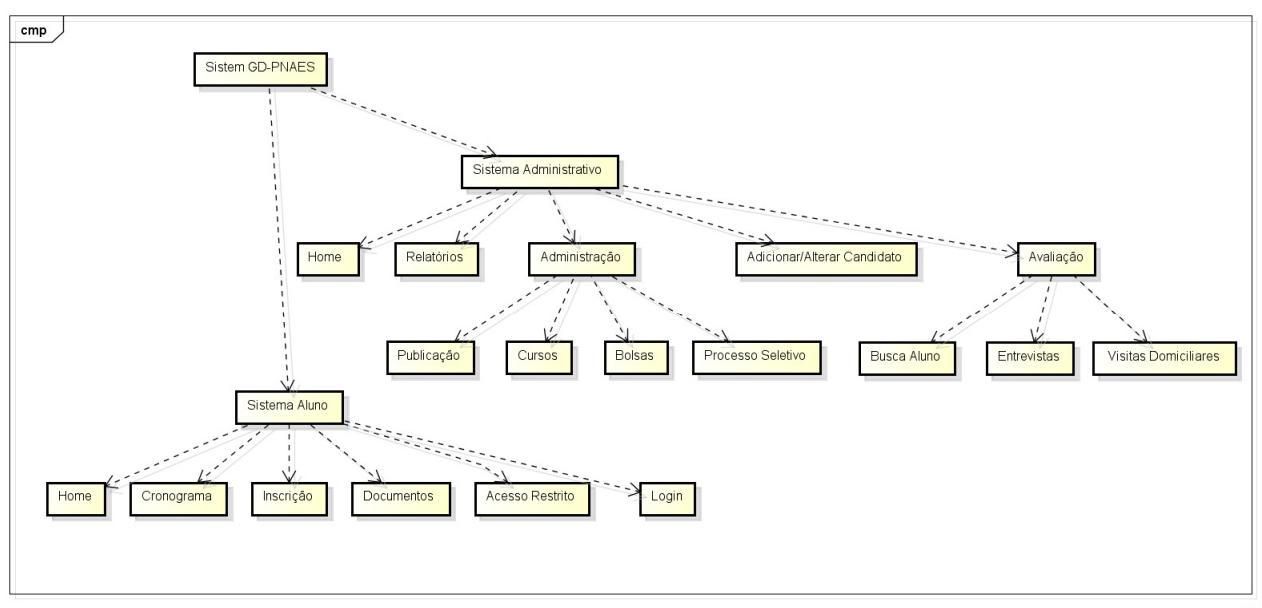

Figura 1: EAP sobre módulos implementados no sistema.

A implantação do sistema se deu por meio da capacitação da Comissão Local de Assistência Estudantil, que replicou o conhecimento aos demais usuários.

A avaliação da qualidade do sistema por meio da visão dos estudantes e da equipe gestora do PNAES. Foram utilizados três mecanismos de avaliação: i) no formulário de inscrição do candidato foi inserida uma pergunta avaliativa; ii) através de formulários impressos aplicados aos alunos durante as entrevistas de seleção, que avaliavam a qualidade de conteúdo, facilidade de navegação, rapidez de acesso e design 
do sistema; e iii) através de um formulário criado no Google Docs aplicado a equipe gestora.

\section{Análise dos Resultados e discussões}

O processo seletivo realizado através do GD-PNAES (Gerenciamento de Distribuição do Programa Nacional de Assistência Estudantil) obteve 402 inscrições, que correspondeu a 53,7 do total de estudantes matriculados na instituição.

Por meio de consulta ao banco de dados, constatou-se que a média de idade dos inscritos foi de 20 anos e que $72,63 \%$ dos candidatos estavam desempregados, indicando o perfil dos candidatos. O tipo de bolsa com maior procura foi Transporte Urbano com 27,61\% do total de inscritos, refletindo uma realidade local relacionada a um problema municipal de falta de ônibus coletivo.

O perfil dos estudantes que procuraram auxílio financeiro através do PNAES foi observado também por meio do curso dos candidatos. O curso Técnico em Vendas ofertado na modalidade de educação de jovens e adultos (EJA) teve um percentual baixo de inscrições $0,74 \%$, enquanto que o curso com maior demanda foi o curso superior de Licenciatura em Computação com $25,62 \%$ de inscrições. A relação do curso com o número de inscrições pode estar relacionado à idade média dos estudantes de cada curso e a habilidade dos estudantes com a informática. Enquanto os estudantes do curso de computação tem facilidade em realizar uma inscrição num sistema web, os alunos da modalidade EJA, têm mais dificuldade e uma média de idade maior.

A avaliação do sistema web implantado, como descrito na metodologia se deu por três formas. A primeira avaliação inserida no formulário de inscrição questionava aos estudantes se o formulário web facilitou a inscrição do candidato. Os resultados mostraram que $49 \%$ responderam que sim, que o sistema web facilitou o processo de inscrição. Já 36\% responderam que havia facilitado, mas que o sistema poderia melhorar ainda. Já $15 \%$ responderam que não, que preferiam fazer a inscrição no formulário impresso.

$\mathrm{Na}$ segunda avaliação do sistema foi realizada com os estudantes selecionados para a segunda fase do processo seletivo do Pnaes, onde identificou-se que 78,84\% dos candidatos já haviam participado dos processos seletivos anteriores, e desses, 50\% afirmaram a melhora do processo seletivo com a utilização do sistema web. Esta avaliação foi aplicada a 52 candidatos onde $55,76 \%$ afirmaram ainda não apresentar dificuldades em fazer a inscrição online e em utilizar o sistema. Outros aspectos do sistema também foram avaliados conforme mostra o Gráfico 2. 


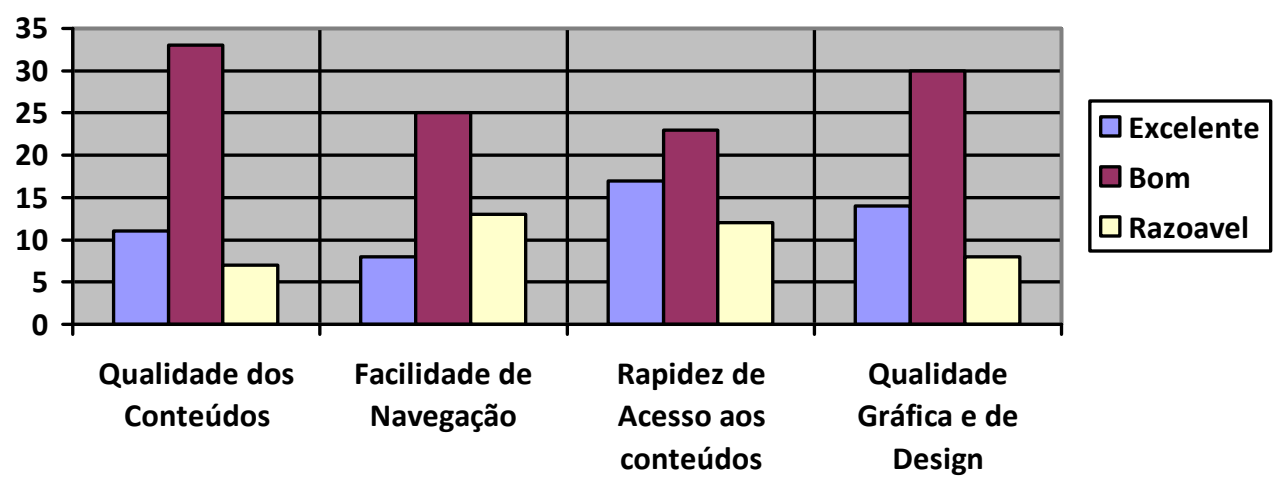

\section{Gráfico 2: Demonstrativo da avaliação pelos alunos.}

A terceira avaliação do sistema foi aplicada a equipe local gestora do PNAES, composta por quatro integrantes. Os resultados podem ser avaliados pelo Gráfico 03.

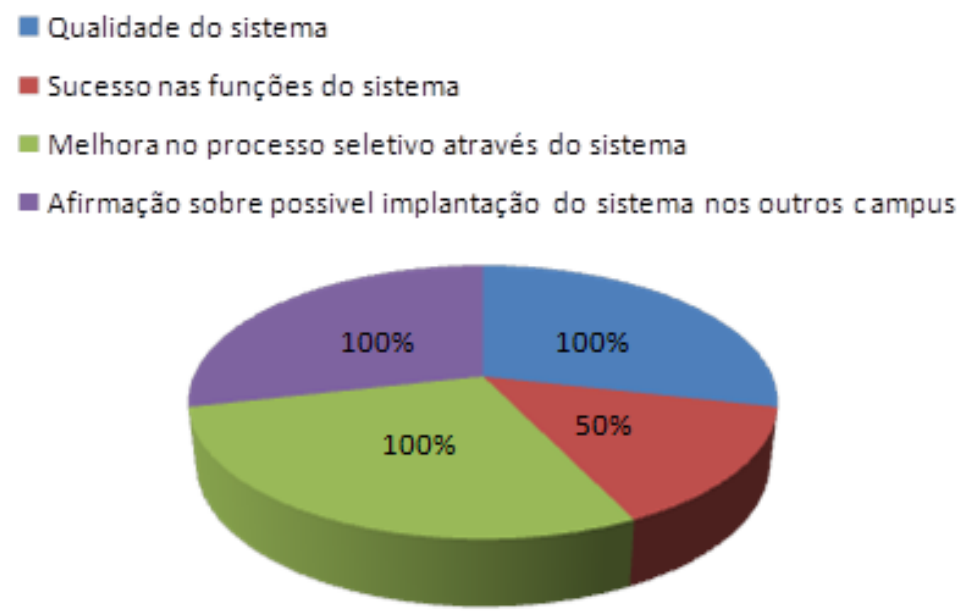

\section{Gráfico 3: Demonstrativo da avaliação da Comissão de Assistência Estudantil.}

O formulário de avaliação analisou o grau de satisfação da equipe gestora que atribuiu a nota oito ao sistema. E num contexto geral a equipe avaliou a implantação do sistema como satisfatória, afirmando ainda que não há dúvidas que sistema GD-PNAES se tornou uma importante ferramenta para o desenvolvimento da Política de Assistência Estudantil no IFTO, Campus Porto Nacional.

O sistema GD-PNAES, trouxe vários avanços para o Campus, consequentemente aos alunos e servidores, pois permitiu aos estudantes e familiares o preenchimento em conjunto do formulário de inscrição "online", além do acesso direto aos editais e documentos necessários para a confirmação das inscrições. Por outro lado, favoreceu a equipe de profissionais do IFTO Campus Porto Nacional a realização de análise dos pedidos de bolsas com maior efetividade, pois antes do sistema, partes dos dados eram lançadas manualmente em uma planilha e levava dias para conclusão dessa etapa. Com a implantação do sistema, esse processo passou a ser feito pelos próprios estudantes, permitindo a equipe acesso direto de todas as informações do candidato por 
meio de relatórios, maior foco na conferência e análise documental e agilidade no processo de seleção dos bolsistas.

Durante a operacionalização oficial do processo seletivo no sistema GD-PNAES, foi possível identificar algumas dificuldades encontradas pelos usuários que podem ser classificadas em dois aspectos. O primeiro atribui-se ao analfabetismo tecnológico no se refere o conhecimento em informática por parte de alguns usuários. Muitos estudantes são oriundos de famílias de baixa renda, não tem acesso à internet e possui pouca habilidade em informática, o que resultou no preenchimento errado de alguns dados durante o processo de inscrição das bolsas ou mesmo falta de alguns deles. $\mathrm{O}$ segundo aspecto, está relacionado à assimilação das perguntas no formulário de inscrição, pois muitos estudantes apresentaram desconhecerem termos como: órgão expedidor, cônjuge, logradouro ou mesmo dados relacionados aos aspectos sociais e econômicos (renda bruta, benefícios etc.). Todos esses fatores foram identificados pela Comissão de Administração do sistema.

\section{Conclusões}

A implantação do sistema GD-PNAES como forma de informatização do processo seletivo para distribuição dos recursos do PNAES foi avaliada de forma positiva, tanto pela equipe gestora do PNAES, quanto pelos estudantes usuários. Ainda que tenha apresentado algumas dificuldades durante a sua execução, os resultados apontados pelas pesquisas avaliativas deixam claro que a proposta inicial foi atingida "contribuir para o desenvolvimento da política estudantil no Campus Porto Nacional no que tange a informatização do processo seletivo das bolsas do Programa Nacional de Assistência Estudantil".

Como afirmado pela equipe gestora do PNAES a implantação do sistema foi satisfatória, e não há dúvidas que sistema GD-PNAES se tornou uma importante ferramenta para o desenvolvimento da Política de Assistência Estudantil no IFTO, Campus Porto Nacional.

Como foi recente a sua implantação, acredita-se que o resultado final seja atingido em médio ou longo prazo. Mas considera-se, um ponto de partida para a informatização deste processo de seleção nos demais campi do IFTO e para o desenvolvimento de novas funcionalidades. Um dos desafios do GD-PNAES consiste na capacidade de avaliação de desempenho do estudante bolsista por meio da integralização com o sistema de registro escolar do Instituto Federal, já que um dos objetivos do PNAES é contribuir para a melhoria do desempenho acadêmico, a partir de medidas que buscam combater situações de repetência e evasão. Outro desafio está na inserção de registro dos atendimentos realizados aos beneficiários do programa pela equipe multidisciplinar.

Uma ferramenta que facilite a execução da assistência estudantil pode torna-la mais capaz para atuar de forma mais planejada não somente como política destinada a suprir carências materiais, mas como um programa social eficaz. 
CBIE-LACLO 2015

Anais do XXI Workshop de Informática na Escola (WIE 2015)

\section{Referências}

Assis, Ana Caroline Lili de. (2013). “ Desafios e possibilidades da política de assistência estudantil da UFJF", Dissertação de Mestrado Profissional em Gestão e Avaliação da Educação Pública. Universidade Federal de Juiz de Fora. Disponível em:<http://www.mestrado.caedufjf.net/wp-content/uploads/2014/03/dissertacao2011-ana-carolina-lili-de-assis.pdf $>$. Acesso em 15 maio 2015.

Garnica, L. A., \& Torkomian, A. L. V. (2009). Gestão de tecnologia em universidades: uma análise do patenteamento e dos fatores de dificuldade e de apoio à transferência de tecnologia no Estado de São Paulo. Gestão \& Produção, 16(4), 624-638.

PORTAL BRASIL (2014). "Mais de R\$ 1 bilhão foram investidos em programas estudantis e melhoria do ensino." Disponível em: < http://www.brasil.gov.br/educacao/2012/09/mais-de-r-1-bi-em-assistencia-estudantilsao-investidos-aos-alunos-e-instituicoes $>$. Acesso em 15 maio 2015.

IFTO (2014).“ Detalhamento das despesas IFTO 2014”. Disponível em: http://www.ifto.edu.br/portal/docs/proad/Or\%C3\%A7amento_IFTO_2014.pdf Acesso em 15 maio 2015.

Project Management Institute - PMI.(2008), "A Guide to the Project Management Body of Knowledge (PMBoK)", 4. ed. PMI Standard - ANSI.

Severino, A. J. (2008). O ensino superior brasileiro: novas configurações e velhos desafios Higher education in Brazil: new configurations and old challenges. P. 73-89.

Tonsig, Sérgio Luiz. (2008), "Engenharia de Software- Análise e Projeto de Sistemas $2^{a}$ ed. Revista e ampliada", Rio de Janeiro: Editora Ciência Moderna Ltda., P. 80 